\title{
Generalized Steffensen's inequality by Montgomery identity
}

\author{
Saad Ihsan Butt ${ }^{1}$, Asfand Fahad ${ }^{2}$, Adil Naseer ${ }^{1 *}$ and Josip Pečarić ${ }^{3}$
}

\section{${ }^{*}$ Correspondence:}

adilnaseer0@gmail.com

1 Department of Mathematics,

COMSATS University Islamabad,

Lahore Campus, Pakistan

Full list of author information is

available at the end of the article

\section{Springer}

\begin{abstract}
By using generalized Montgomery identity and Green functions we proved several identities which assist in developing connections with Steffensen's inequality. Under the assumptions of $n$-convexity and $n$-concavity many inequalities, which generalize Steffensen's inequality, inequalities from (Fahad et al. in J. Math. Inequal. 9:481-487, 2015; Pečarić in Southeast Asian Bull. Math. 13:89-91, 1989; Rabier in Proc. Am. Math. Soc. 140:665-675, 2012), and their reverse, have been proved. Generalization of some inequalities (and their reverse) which are related to Hardy-type inequality (Fahad et al. in J. Math. Inequal. 9:481-487, 2015) have also been proved. New bounds of Ostrowski and Grüss type inequalities have been developed. Moreover, we formulate generalized Steffensen-type linear functionals and prove their monotonicity for the generalized class of $(n+1)$-convex functions at a point. At the end, we present some applications of our study to the theory of exponentially convex functions.
\end{abstract}

MSC: Primary 26D10; secondary 26D20

Keywords: Steffensen's inequality; Green's function; Montgomery's identity; $(n+1)$-convex function at a point

\section{Introduction}

The key in the solutions of many problems in mathematics is somehow related to an inequality or an equivalent notion. Mathematical inequalities are also being used in other branches of science, for example, contributions in physics and chemistry can be seen in [9] and [10], respectively. Integral inequalities such as Hardy's inequality, Steffensen's inequality, and Ostrowski's inequality have been topics of interest of many mathematicians since their pronouncement. Several generalizations of these inequalities have been proved for convex functions, beta $m$-convex functions, $n$-convex functions, and other classes of functions, for example, see $[4,7,12,16]$, and [17]. Steffensen's inequality was proved in [18]: if $\psi, f:[c, d] \rightarrow \mathbb{R}$, with $\psi$ being a decreasing function and function $f$ having range in $[0,1]$, then

$$
\int_{c}^{d} \psi(z) f(z) d t \leq \int_{c}^{c+\theta} \psi(z) d z, \quad \text { where } \theta=\int_{c}^{d} f(z) d z
$$

A massive literature body dealing with several variants and improvements of (1) can be seen in $[14,16]$ and the references therein. Pečarić [13] gave a nice generalization of (1) as follows.

(c) The Author(s) 2019. This article is distributed under the terms of the Creative Commons Attribution 4.0 International License (http://creativecommons.org/licenses/by/4.0/), which permits unrestricted use, distribution, and reproduction in any medium, provided you give appropriate credit to the original author(s) and the source, provide a link to the Creative Commons license, and indicate if changes were made. 
Theorem 1 For two increasing functions $\psi$ on an interval $J$ and $f$ on $[c, d]$ with $\{c, d, f(c)$, $f(d)\} \subseteq J$ and $f$ differentiable, we have

(i) If $f(t) \leq t$, then

$$
\int_{f(c)}^{f(d)} \psi(z) d z \leq \int_{c}^{d} \psi(z) f^{\prime}(z) d z
$$

(ii) If $f(t) \geq t$, then (2) holds in reverse direction.

Remark 1 We can consider $f$ to be absolute continuous instead of differentiable function and the suppositions of Theorem 1 can also be weakened. In fact, for an increasing function $\psi$, the function $\Psi(x)=\int_{c}^{x} \psi(z) d z$ is well defined and satisfies $\Psi^{\prime}=\psi$ at all except the set of points with measure zero. One can substitute $x=f(z)$ in (2) (see [6, Corollary 20.5]) provided that $f$ is an absolutely continuous increasing function; consequently,

$$
\Psi(f(d))-\Psi(f(c))=\int_{f(c)}^{f(d)} \psi(x) d x=\int_{c}^{d} \psi(f(z)) f^{\prime}(z) d z \leq \int_{c}^{d} \psi(z) f^{\prime}(z) d z,
$$

where the last inequality holds when $f(z) \leq z$.

Another generalization has been established by Rabier [17].

Theorem 2 Let $\psi$ be continuous and convex on $[0, \infty)$ with $\psi(0)=0$. If $h$ is non-negative and bounded almost everywhere on $(0, b)$ with $\|h\|_{\infty} \leq 1$, then $h \psi^{\prime}$ is integrable on $(0, b)$ and

$$
\psi\left(\int_{0}^{b} h(t) d t\right) \leq \int_{0}^{b} h(t) \psi^{\prime}(t) d t
$$

In [4], substitutions presented conclude that from (3) one may get all (1), (2), and (4). Recently, Fahad, Pečarić, and Praljak proved generalization [4] of (1) by extending the results given in [13].

Now, we include some important conclusions given in [4].

Corollary 1 Suppose that $\psi: J \rightarrow \mathbb{R}, f:[c, d] \rightarrow \mathbb{R}$ are two differentiable functions with $f$ non-decreasing as well, where J is an interval containing $[c, d], f(c)$, and $f(d)$. If $\psi$ is convex, then:

(i) Iff $(t) \leq t$, then

$$
\psi(f(d)) \leq \psi(f(c))+\int_{c}^{d} \psi^{\prime}(z) f^{\prime}(z) d z
$$

(ii) If $f(t) \geq t$, then (5) holds in reverse direction.

Corollary 1 gives (3) and therefore leads to (1), (2), and (4). Next we narrate some further important results of [4].

Corollary 2 Let $\psi:[0, d] \rightarrow \mathbb{R}$ be a convex, differentiable function with $\psi(0)=0$ and $f$ be a non-negative function on $[0, d]$. 
(i) If $\int_{0}^{t} f(z) d z \leq t$ for every $t \in[0, d]$, then

$$
\psi\left(\int_{0}^{d} f(z) d z\right) \leq \int_{0}^{d} \psi^{\prime}(z) f(z) d z
$$

(ii) If $t \leq \int_{0}^{t} f(z) d z$ for every $t \in[0, d]$, then (6) holds reversely.

Corollary 3 Consider $\psi$ and $f$ be as defined in Corollary 2 , and let $\lambda:[0, d] \rightarrow[0,+\infty)$ and denote $\Lambda(z)=\int_{z}^{d} \lambda(t) d t$.

(i) If $\int_{0}^{t} f(z) d z \leq t$ for every $t \in[0, d]$, then

$$
\int_{0}^{d} \lambda(t) \psi\left(\int_{0}^{t} f(z) d z\right) d t \leq \int_{0}^{d} \Lambda(z) \psi^{\prime}(z) f(z) d z .
$$

(ii) If $t \leq \int_{0}^{t} f(z) d z$ for every $t \in[0, d]$, then (7) holds reversely.

The main objective of this article is to establish generalization of (3) and ultimately produce the generalizations of (1), (2), and (4). The connection between classical Hardy-type inequalities and inequalities (6) and (7) has been elaborated in [4]. Due to significance of (6) and (7), we prove their generalizations as well. As an application, we present contribution of new inequalities to theory of exponentially convex functions and $(n+1)$-convex functions at a point. We use Čebyšev functional to construct new bounds of Grüss and Ostrowski type inequalities. To achieve this objective, we use the generalized Montgomery identity and Green functions. The following two lemmas will be useful in our construction as well, see [11] and [5].

Lemma 1 For a function $\psi \in C^{2}([c, d])$, we have:

$$
\begin{aligned}
& \psi(\xi)=\frac{d-\xi}{d-c} \psi(c)+\frac{\xi-c}{d-c} \psi(d)+\int_{c}^{d} G_{*, 1}(\xi, u) \psi^{\prime \prime}(u) d u, \\
& \psi(\xi)=\psi(c)+(\xi-c) \psi^{\prime}(d)+\int_{c}^{d} G_{*, 2}(\xi, u) \psi^{\prime \prime}(u) d u, \\
& \psi(\xi)=\psi(d)+(d-\xi) \psi^{\prime}(c)+\int_{c}^{d} G_{*, 3}(\xi, u) \psi^{\prime \prime}(u) d u, \\
& \psi(\xi)=\psi(d)-(d-c) \psi^{\prime}(d)+(\xi-c) \psi^{\prime}(c)+\int_{c}^{d} G_{*, 4}(\xi, u) \psi^{\prime \prime}(u) d u, \\
& \psi(\xi)=\psi(c)+(d-c) \psi^{\prime}(c)-(d-\xi) \psi^{\prime}(d)+\int_{c}^{d} G_{*, 5}(\xi, u) \psi^{\prime \prime}(u) d u,
\end{aligned}
$$

where

$$
\begin{aligned}
& G_{*, 1}(\xi, u)= \begin{cases}\frac{(\xi-d)(u-c)}{d-c}, & \text { if } c \leq u \leq \xi, \\
\frac{(u-d)(\xi-c)}{d-c}, & \text { if } \xi<u \leq d,\end{cases} \\
& G_{*, 2}(\xi, u)= \begin{cases}c-u, & \text { if } c \leq u \leq \xi, \\
c-\xi, & \text { if } \xi<u \leq d,\end{cases}
\end{aligned}
$$




$$
\begin{gathered}
G_{*, 3}(\xi, u)= \begin{cases}\xi-d, & \text { if } c \leq u \leq \xi, \\
u-d, & \text { if } \xi<u \leq d,\end{cases} \\
G_{*, 4}(\xi, u)= \begin{cases}\xi-c, & \text { if } c \leq u \leq \xi, \\
u-c, & \text { if } \xi<u \leq d,\end{cases}
\end{gathered}
$$

and

$$
G_{*, 5}(\xi, u)= \begin{cases}d-u, & \text { if } c \leq u \leq \xi \\ d-\xi, & \text { if } \xi<u \leq d\end{cases}
$$

Lemma 2 Let $\psi \in C^{1}[c, d]$, then

$$
\begin{aligned}
& \psi(\xi)=\frac{1}{d-c} \int_{c}^{d} \psi(u) d u+\int_{c}^{d} p_{1}(\xi, u) \psi^{\prime}(u) d u, \\
& \psi(\xi)=\psi(d)+\int_{c}^{d} p_{2}(\xi, u) \psi^{\prime}(u) d u,
\end{aligned}
$$

and

$$
\psi(\xi)=\psi(c)+\int_{c}^{d} p_{3}(\xi, u) \psi^{\prime}(u) d u
$$

where

$$
\begin{aligned}
& p_{1}(\xi, u)= \begin{cases}\frac{u-c}{d-c}, & \text { if } c \leq u \leq \xi, \\
\frac{u-d}{d-c}, & \text { if } \xi<u \leq d,\end{cases} \\
& p_{2}(\xi, u)= \begin{cases}0, & \text { if } c \leq u \leq \xi, \\
-1, & \text { if } \xi<u \leq d,\end{cases} \\
& p_{3}(\xi, u)= \begin{cases}1, & \text { if } c \leq u \leq \xi, \\
0, & \text { if } \xi<u \leq d .\end{cases}
\end{aligned}
$$

Clearly,

$$
\begin{aligned}
& p_{i}(\xi, u)=\frac{\partial G_{*, i}(\xi, u)}{\partial \xi} \quad \text { for all } i=1,2,3 \\
& p_{2}(\xi, u)=\frac{\partial G_{*, 5}(\xi, u)}{\partial \xi}, \quad \text { and } \quad p_{3}(\xi, u)=\frac{\partial G_{*, 4}(\xi, u)}{\partial \xi} .
\end{aligned}
$$

From now on, during the calculations, we will use $p_{i}(\xi, u)$ corresponding to $\frac{\partial G_{*, i}(\xi, u)}{\partial \xi}$ for $i=1,2,3$ and for $\frac{\partial G_{*, 4}(\xi, u)}{\partial \xi}, \frac{\partial G_{*, 5}(\xi, u)}{\partial \xi}$ we use $p_{3}(\xi, s)$ and $p_{2}(\xi, s)$ respectively.

To prove our main results in the next section, we also use the following generalized Montgomery identity via Taylor's formula given in [1]. 
Theorem 3 Let $n \in \mathbb{N}, \psi: J \rightarrow \mathbb{R}$ be such that $\psi^{(n-1)}$ is absolutely continuous, $J \subset \mathbb{R}$ be an open interval, $c, d \in J, c<d$. Then

$$
\begin{aligned}
\psi(s)= & \frac{1}{d-c} \int_{c}^{d} \psi(\xi) d \xi+\sum_{l=0}^{n-2} \frac{\psi^{(l+1)}(c)}{l !(l+2)} \frac{(s-c)^{l+2}}{d-c}-\sum_{l=0}^{n-2} \frac{\psi^{(l+1)}(d)}{l !(l+2)} \frac{(s-d)^{l+2}}{d-c} \\
& +\frac{1}{(n-1) !} \int_{c}^{d} R_{n}(s, t) \psi^{(n)}(t) d t,
\end{aligned}
$$

where

$$
R_{n}(s, t)= \begin{cases}-\frac{(s-t)^{n}}{n(d-c)}+\frac{s-c}{d-c}(s-t)^{n-1}, & c \leq t \leq s \\ -\frac{(s-t)^{n}}{n(d-c)}+\frac{s-d}{d-c}(s-t)^{n-1}, & s<t \leq d .\end{cases}
$$

The next section contains the main result of the article.

\section{Generalization of Steffensen's inequality by generalized Montgomery identity}

For our convenience, we use the following notations and assumptions:

$$
\begin{aligned}
& \mathbb{S}_{1}(\psi, f, c, d)=\psi(f(c))+\int_{c}^{d} \psi^{\prime}(z) f^{\prime}(z) d z-\psi(f(d)), \\
& \mathbb{S}_{2}(\psi, f, d)=\int_{0}^{d} \psi^{\prime}(z) f(z) d z-\psi\left(\int_{0}^{d} f(z) d z\right), \\
& \mathbb{S}_{3}(\psi, f, \lambda, d)=\int_{0}^{d} \Lambda(z) \psi^{\prime}(z) f(z) d z-\int_{0}^{d} \lambda(t) \psi\left(\int_{0}^{t} f(z) d z\right) d t .
\end{aligned}
$$

$\left(A_{1}\right)$ For $n \in \mathbb{N}, n \geq 3$, let $\psi:[c, d] \rightarrow \mathbb{R}$ be an $n$ times differentiable function with $\psi^{(n-1)}$ absolutely continuous on $[c, d]$.

$\left(A_{2}\right)$ For $n \in \mathbb{N}, n \geq 3$, let $\psi:[0, d] \rightarrow \mathbb{R}$ be an $n$ times differentiable function with $\psi(0)=$ 0 and $\psi^{(n-1)}$ absolutely continuous on $[0, d]$.

The first part of this section is the generalization of (5). For this, we start with the following theorem.

Theorem 4 Consider $\left(A_{1}\right)$ with $f$ be as in Corollary $1(i)$ and $R_{n}$ be defined by (26), then:

(a) For $j=1,2,4,5$, we have

$$
\begin{aligned}
\mathbb{S}_{1}(\psi, f, c, d) & \\
= & \frac{\psi^{\prime}(c)-\psi^{\prime}(d)}{d-c} \int_{c}^{d} \mathbb{S}_{1}\left(G_{*, j}(\cdot, s), f, c, d\right) d s \\
& +\frac{1}{d-c} \int_{c}^{d} \mathbb{S}_{1}\left(G_{*, j}(\cdot, s), f, c, d\right) \\
& \times\left(\sum_{l=2}^{n-1} \frac{l}{(l-1) !}\left(\psi^{(l)}(c)(s-c)^{l-1}-\psi^{(l)}(d)(s-d)^{l-1}\right)\right) d s \\
& +\frac{1}{(n-3) !} \int_{c}^{d} \psi^{(n)}(v)\left(\int_{c}^{d} \mathbb{S}_{1}\left(G_{*, j}(\cdot, s), f, c, d\right) \tilde{R}_{n-2}(s, v) d s\right) d v .
\end{aligned}
$$


(b) If $\psi^{\prime}(c)=0$, then

$$
\begin{aligned}
& \mathbb{S}_{1}(\psi, f, c, d) \\
& =\frac{\psi^{\prime}(c)-\psi^{\prime}(d)}{d-c} \int_{c}^{d} \mathbb{S}_{1}\left(G_{*, 3}(\cdot, s), f, c, d\right) d s \\
& \quad+\frac{1}{d-c} \int_{c}^{d} \mathbb{S}_{1}\left(G_{*, 3}(\cdot, s), f, c, d\right) \\
& \quad \times\left(\sum_{l=2}^{n-1} \frac{l}{(l-1) !}\left(\psi^{(l)}(c)(s-c)^{l-1}-\psi^{(l)}(d)(s-d)^{l-1}\right)\right) d s \\
& \quad+\frac{1}{(n-3) !} \int_{c}^{d} \psi^{(n)}(v)\left(\int_{c}^{d} \mathbb{S}_{1}\left(G_{*, 3}(\cdot, s), f, c, d\right) \tilde{R}_{n-2}(s, v) d s\right) d v,
\end{aligned}
$$

where

$$
\tilde{R}_{n-2}(s, v)= \begin{cases}\frac{1}{d-c}\left[\frac{(s-v)^{n-2}}{(n-2)}+(s-c)(s-v)^{n-3}\right], & c \leq v \leq s \\ \frac{1}{d-c}\left[\frac{(s-v)^{n-2}}{(n-2)}+(s-d)(s-v)^{n-3}\right], & s<v \leq d\end{cases}
$$

and

( $\left.a^{\prime}\right)$ For $j=1,2,4,5$, we have

$$
\begin{aligned}
\mathbb{S}_{1}(\psi, f, c, d) & \left(\frac{\psi^{\prime}(d)-\psi^{\prime}(c)}{d-c}\right) \int_{c}^{d} \mathbb{S}_{1}\left(G_{*, j}(\cdot, s), f, c, d\right) d s \\
& +\frac{1}{d-c} \int_{c}^{d} \mathbb{S}_{1}\left(G_{*, j}(\cdot, s), f, c, d\right) \\
& \times\left(\sum_{l=3}^{n-1} \frac{\psi^{(l)}(c)(s-c)^{l-1}-\psi^{(l)}(d)(s-d)^{l-1}}{(l-3) !(l-1)}\right) d s \\
& +\frac{1}{(n-3) !} \int_{c}^{d} \psi^{(n)}(v)\left(\int_{c}^{d} \mathbb{S}_{1}\left(G_{*, j}(\cdot, s), f, c, d\right) R_{n-2}(s, v) d s\right) d v .
\end{aligned}
$$

(b') If $\psi^{\prime}(c)=0$, then

$$
\begin{aligned}
& \mathbb{S}_{1}(\psi, f, c, d) \\
& =\left(\frac{\psi^{\prime}(d)-\psi^{\prime}(c)}{d-c}\right) \int_{c}^{d} \mathbb{S}_{1}\left(G_{*, 3}(\cdot, s), f, c, d\right) d s \\
& \quad+\frac{1}{d-c} \int_{c}^{d} \mathbb{S}_{1}\left(G_{*, 3}(\cdot, s), f, c, d\right)\left(\sum_{l=3}^{n-1} \frac{\psi^{(l)}(c)(s-c)^{l-1}-\psi^{(l)}(d)(s-d)^{l-1}}{(l-3) !(l-1)}\right) d s \\
& \quad+\frac{1}{(n-3) !} \int_{c}^{d} \psi^{(n)}(v)\left(\int_{c}^{d} \mathbb{S}_{1}\left(G_{*, 3}(\cdot, s), f, c, d\right) R_{n-2}(s, v) d s\right) d v .
\end{aligned}
$$

Proof By using (8) and (18) for $\psi$ and $\psi^{\prime}$ respectively and then applying (24), we get

$$
\mathbb{S}_{1}(\psi, f, c, d)=\int_{c}^{d} \mathbb{S}_{1}\left(G_{*, j}(\cdot, s), f, c, d\right) \psi^{\prime \prime}(s) d s .
$$


Differentiating (25) twice with respect to first variable, we have

$$
\begin{aligned}
\psi^{\prime \prime}(s)= & \frac{\psi^{\prime}(c)-\psi^{\prime}(d)}{d-c}+\sum_{l=2}^{n-1}\left(\frac{l}{(l-1) !}\right)\left(\frac{\psi^{(l)}(c)(s-c)^{l-1}-\psi^{(l)}(d)(s-d)^{l-1}}{d-c}\right) \\
& +\frac{1}{(n-3) !} \int_{c}^{d} \tilde{R}_{n-2}(s, v) \psi^{(n)}(v) d v .
\end{aligned}
$$

Using (32) in (31), we get

$$
\begin{aligned}
& \mathbb{S}_{1}(\psi, f, c, d) \\
& =\frac{\psi^{\prime}(c)-\psi^{\prime}(d)}{d-c} \int_{c}^{d} \mathbb{S}_{1}\left(G_{*, 1}(\cdot, s), f, c, d\right) d s \\
& \quad+\sum_{l=2}^{n-1}\left(\frac{l}{(l-1) !}\right) \int_{c}^{d} \mathbb{S}_{1}\left(G_{*, 1}(\cdot, s), f, c, d\right)\left(\frac{\psi^{(l)}(c)(s-c)^{l-1}-\psi^{(l)}(d)(s-d)^{l-1}}{d-c}\right) d s \\
& \quad+\frac{1}{(n-3) !} \int_{c}^{d} \mathbb{S}_{1}\left(G_{*, 1}(\cdot, s), f, c, d\right)\left(\int_{a}^{b} \tilde{R}_{n-2}(s, v) \psi^{(n)}(v) d v\right) d s .
\end{aligned}
$$

By applying Fubini's theorem in the last term, we have (27). Next, using formula (25) on the function $\psi^{\prime \prime}$, replacing $n$ by $n-2(n \geq 3)$, and rearranging the indices, we have

$$
\begin{aligned}
\psi^{\prime \prime}(s)= & \left(\frac{\psi^{\prime}(d)-\psi^{\prime}(c)}{d-c}\right) \\
& +\sum_{l=3}^{n-1}\left(\frac{1}{(l-3) !(l-1)}\right)\left(\frac{\psi^{(l)}(c)(s-c)^{l-1}-\psi^{(l)}(b)(s-d)^{l-1}}{d-c}\right) \\
& +\frac{1}{(n-3) !} \int_{c}^{d} R_{n-2}(s, v) \psi^{(n)}(v) d v .
\end{aligned}
$$

Similarly, using (33) in (31) and applying Fubini's theorem, we get (30). The proof for $j=$ $2,3,4,5$ can be obtained in a similar way except for the use of (9)-(12) and (19)-(20).

From the next two theorems, we get generalization of Steffensen's inequality and its reverse by generalizing (5) and its reverse.

Theorem 5 Consider $\left(A_{1}\right)$ with $f$ be as in Corollary $1(i)$, and let $R_{n}, \tilde{R}_{n-2}$ be as in Theorem 4 . If $\psi$ is n-convex and

$$
\int_{c}^{d} \mathbb{S}_{1}\left(G_{*, j}(\cdot, s), f, c, d\right) \tilde{R}_{n-2}(s, v) d s \geq 0,
$$

then

$$
\begin{aligned}
\mathbb{S}_{1}(\psi, f, c, d) \geq & \frac{\psi^{\prime}(c)-\psi^{\prime}(d)}{d-c} \int_{c}^{d} \mathbb{S}_{1}\left(G_{*, j}(\cdot, s), f, c, d\right) d s \\
& +\frac{1}{d-c} \int_{c}^{d} \mathbb{S}_{1}\left(G_{*, j}(\cdot, s), f, c, d\right) \\
& \times\left(\sum_{l=2}^{n-1} \frac{l}{(l-1) !}\left(\psi^{(l)}(c)(s-c)^{l-1}-\psi^{(l)}(d)(s-d)^{l-1}\right)\right) d s
\end{aligned}
$$


and if

$$
\int_{c}^{d} \mathbb{S}_{1}\left(G_{*, j}(\cdot, s), f, c, d\right) R_{n-2}(s, v) d s \geq 0
$$

then

$$
\begin{aligned}
& \mathbb{S}_{1}(\psi, f, c, d) \\
& \geq\left(\frac{\psi^{\prime}(d)-\psi^{\prime}(c)}{d-c}\right) \int_{c}^{d} \mathbb{S}_{1}\left(G_{*, j}(\cdot, s), f, c, d\right) d s \\
& \quad+\frac{1}{d-c} \int_{c}^{d} \mathbb{S}_{1}\left(G_{*, j}(\cdot, s), f, c, d\right)\left(\sum_{l=3}^{n-1} \frac{\psi^{(l)}(c)(s-c)^{l-1}-\psi^{(l)}(d)(s-d)^{l-1}}{(l-3) !(l-1)}\right) d s
\end{aligned}
$$

for $j=1,2, \ldots, 5$, where $\psi^{\prime}(c)=0$ for $j=3$.

Proof Since $\psi^{(n-1)}$ is absolutely continuous on $[c, d], \psi^{(n)}$ exists almost everywhere. As $\psi$ is $n$-convex, so $\psi^{(n)}(x) \geq 0$ for all $x \in[c, d]$ (see [16], p. 16). Hence we can apply Theorem 4 to obtain (35) and (37) respectively.

Theorem 6 Consider $\left(A_{1}\right)$ with $f$ be as in Corollary $1(i)$, and let $R_{n}, \tilde{R}_{n-2}$ be as in Theorem 4 . If $\psi$ is n-convex, then:

(i) If $n$ is even and $n \geq 4$, then (35) holds.

(ii) Let inequality (35) be satisfied and

$$
\sum_{l=1}^{n-1} \frac{l}{(l-1) !}\left(\psi^{(l)}(c)(s-c)^{l-1}-\psi^{(l)}(d)(s-d)^{l-1}\right) \geq 0 ; \quad \forall s \in[c, d],
$$

OR

(37) be satisfied and

$$
\psi^{\prime}(d)-\psi^{\prime}(c)+\sum_{l=3}^{n-1} \frac{\psi^{(l)}(c)(s-c)^{l-1}-\psi^{(l)}(d)(s-d)^{l-1}}{(l-3) !(l-1)} \geq 0 \quad \forall s \in[c, d]
$$

then we have

$$
\mathbb{S}_{1}(\psi, f, c, d) \geq 0
$$

Proof Since Green's function $G$ is convex and $f$ is as in Corollary 1(a), therefore, $S_{1}\left(G_{*, j}(\cdot, s), f, c, d\right) \geq 0$ holds by virtue of Corollary $1(a)$. Moreover, $\tilde{R}_{n-2}(s, v) \geq 0$ for $n=4,6, \ldots$, so (34) holds. As $\psi$ is $n$-convex, hence by Theorem 5 , we obtain (35). Further, by using (38) in (35) or (39) in (37), we have (40).

Remark 2 Inequalities (35) and (37) hold in reverse directions if either the inequalities in (34) and (36) are reversed or $-\psi$ is $n$-convex. By using these reverse inequalities and applying a similar technique as in Theorem 6 , one may prove $\mathbb{S}_{1}(\psi, f, c, d) \leq 0$, which gives the reverse of (1) inequality. 
In the next theorem, we prove a few identities which enable us to prove generalization of (6) and its reverse.

Theorem 7 Consider $\left(A_{2}\right)$ and let $f$ be as in Corollary 2( $\left.i\right)$, then:

(a)

$$
\begin{aligned}
\mathbb{S}_{2}(\psi, f, d) & \\
= & \frac{\psi^{\prime}(0)-\psi^{\prime}(d)}{d} \int_{0}^{d} \mathbb{S}_{2}\left(G_{*, j}(\cdot, s), f, d\right) d s \\
& +\frac{1}{d} \int_{0}^{d} \mathbb{S}_{2}\left(G_{*, j}(\cdot, s), f, d\right)\left(\sum_{l=2}^{n-1} \frac{l}{(l-1) !}\left(\psi^{(l)}(0)(s)^{l-1}-\psi^{(l)}(d)(s-d)^{l-1}\right)\right) d s \\
& +\frac{1}{(n-3) !} \int_{0}^{d} \psi^{(n)}(v)\left(\int_{0}^{d} \mathbb{S}_{2}\left(G_{*, j}(\cdot, s), f, d\right) \tilde{R}_{0, n-2}(s, v) d s\right) d v
\end{aligned}
$$

for $j=1,2$.

(b) If $\psi^{\prime}(0)=0$, then

$$
\begin{aligned}
\mathbb{S}_{2}(\psi, f, d)+\psi(d) & \\
= & \frac{\psi^{\prime}(0)-\psi^{\prime}(d)}{d} \int_{0}^{d} \mathbb{S}_{2}\left(G_{*, 3}(\cdot, s), f, d\right) d s \\
& +\frac{1}{d} \int_{0}^{d} \mathbb{S}_{2}\left(G_{*, 3}(\cdot, s), f, d\right)\left(\sum_{l=2}^{n-1} \frac{l}{(l-1) !}\left(\psi^{(l)}(0)(s)^{l-1}-\psi^{(l)}(d)(s-d)^{l-1}\right)\right) d s \\
& +\frac{1}{(n-3) !} \int_{0}^{d} \psi^{(n)}(v)\left(\int_{0}^{d} \mathbb{S}_{2}\left(G_{*, 3}(\cdot, s), f, d\right) \tilde{R}_{0, n-2}(s, v) d s\right) d v .
\end{aligned}
$$

(c)

$$
\begin{aligned}
\mathbb{S}_{2}(\psi, f, d)+\psi(d)-d \psi^{\prime}(d) \\
=\frac{\psi^{\prime}(0)-\psi^{\prime}(d)}{d} \int_{0}^{d} \mathbb{S}_{2}\left(G_{*, 4}(\cdot, s), f, d\right) d s \\
\quad+\frac{1}{d} \int_{0}^{d} \mathbb{S}_{2}\left(G_{*, 4}(\cdot, s), f, d\right)\left(\sum_{l=2}^{n-1} \frac{l}{(l-1) !}\left(\psi^{(l)}(0)(s)^{l-1}-\psi^{(l)}(d)(s-d)^{l-1}\right)\right) d s \\
\quad+\frac{1}{(n-3) !} \int_{0}^{d} \psi^{(n)}(v)\left(\int_{0}^{d} \mathbb{S}_{2}\left(G_{*, 4}(\cdot, s), f, d\right) \tilde{R}_{0, n-2}(s, v) d s\right) d v .
\end{aligned}
$$

(d) If $\psi^{\prime}(0)=0$, then

$$
\begin{aligned}
& \mathbb{S}_{2}(\psi, f, d)-d \psi^{\prime}(d) \\
& \quad=\frac{\psi^{\prime}(0)-\psi^{\prime}(d)}{d} \int_{0}^{d} \mathbb{S}_{2}\left(G_{*, 5}(\cdot, s), f, d\right) d s
\end{aligned}
$$




$$
\begin{aligned}
& +\frac{1}{d} \int_{0}^{d} \mathbb{S}_{2}\left(G_{*, 5}(\cdot, s), f, d\right)\left(\sum_{l=2}^{n-1} \frac{l}{(l-1) !}\left(\psi^{(l)}(0)(s)^{l-1}-\psi^{(l)}(d)(s-d)^{l-1}\right)\right) d s \\
& +\frac{1}{(n-3) !} \int_{0}^{d} \psi^{(n)}(v)\left(\int_{0}^{d} \mathbb{S}_{2}\left(G_{*, 5}(\cdot, s), f, d\right) \tilde{R}_{0, n-2}(s, v) d s\right) d v,
\end{aligned}
$$

where $\tilde{R}_{0, n-2}$ is obtained by taking $c=0$ in (29), and

$\left(a^{\prime}\right)$

$$
\begin{aligned}
\mathbb{S}_{2}(\psi, f, d)= & \left(\frac{\psi^{\prime}(d)-\psi^{\prime}(0)}{d}\right) \int_{0}^{d} \mathbb{S}_{2}\left(G_{*, j}(\cdot, s), f, d\right) d s \\
& +\frac{1}{d} \int_{0}^{d} \mathbb{S}_{2}\left(G_{*, j}(\cdot, s), f, d\right)\left(\sum_{l=3}^{n-1} \frac{\psi^{(l)}(0)(s)^{l-1}-\psi^{(l)}(d)(s-d)^{l-1}}{(l-3) !(l-1)}\right) d s \\
& +\frac{1}{(n-3) !} \int_{0}^{d} \psi^{(n)}(v)\left(\int_{0}^{d} \mathbb{S}_{2}\left(G_{*, j}(\cdot, s), f, d\right) R_{0, n-2}(s, v) d s\right) d v
\end{aligned}
$$

for $j=1,2$.

$\left(b^{\prime}\right)$ If $\psi^{\prime}(0)=0$, then

$$
\begin{aligned}
\mathbb{S}_{2}(\psi, f, d)+\psi(d) \\
=\left(\frac{\psi^{\prime}(d)-\psi^{\prime}(0)}{d}\right) \int_{0}^{d} \mathbb{S}_{2}\left(G_{*, 3}(\cdot, s), f, d\right) d s \\
\quad+\frac{1}{d} \int_{0}^{d} \mathbb{S}_{2}\left(G_{*, 3}(\cdot, s), f, d\right)\left(\sum_{l=3}^{n-1} \frac{\psi^{(l)}(0)(s)^{l-1}-\psi^{(l)}(d)(s-d)^{l-1}}{(l-3) !(l-1)}\right) d s \\
\quad+\frac{1}{(n-3) !} \int_{0}^{d} \psi^{(n)}(v)\left(\int_{0}^{d} \mathbb{S}_{2}\left(G_{*, 3}(\cdot, s), f, d\right) R_{0, n-2}(s, v) d s\right) d v .
\end{aligned}
$$

$\left(c^{\prime}\right)$

$$
\begin{aligned}
\mathbb{S}_{2}(\psi, f, d)+\psi(d)-d \psi^{\prime}(d) \\
=\left(\frac{\psi^{\prime}(d)-\psi^{\prime}(0)}{d}\right) \int_{0}^{d} \mathbb{S}_{2}\left(G_{*, 4}(\cdot, s), f, d\right) d s \\
\quad+\frac{1}{d} \int_{0}^{d} \mathbb{S}_{2}\left(G_{*, 4}(\cdot, s), f, d\right)\left(\sum_{l=3}^{n-1} \frac{\psi^{(l)}(0)(s)^{l-1}-\psi^{(l)}(d)(s-d)^{l-1}}{(l-3) !(l-1)}\right) d s \\
\quad+\frac{1}{(n-3) !} \int_{0}^{d} \psi^{(n)}(v)\left(\int_{0}^{d} \mathbb{S}_{2}\left(G_{*, 4}(\cdot, s), f, d\right) R_{0, n-2}(s, v) d s\right) d v .
\end{aligned}
$$

(d') If $\psi^{\prime}(0)=0$, then

$$
\begin{aligned}
& \mathbb{S}_{2}(\psi, f, d)-d \psi^{\prime}(d) \\
& \quad=\left(\frac{\psi^{\prime}(d)-\psi^{\prime}(0)}{d}\right) \int_{0}^{d} \mathbb{S}_{2}\left(G_{*, 5}(\cdot, s), f, d\right) d s
\end{aligned}
$$




$$
\begin{aligned}
& +\frac{1}{d} \int_{0}^{d} \mathbb{S}_{2}\left(G_{*, 5}(\cdot, s), f, d\right)\left(\sum_{l=3}^{n-1} \frac{\psi^{(l)}(0)(s)^{l-1}-\psi^{(l)}(d)(s-d)^{l-1}}{(l-3) !(l-1)}\right) d s \\
& +\frac{1}{(n-3) !} \int_{0}^{d} \psi^{(n)}(v)\left(\int_{0}^{d} \mathbb{S}_{2}\left(G_{*, 5}(\cdot, s), f, d\right) R_{0, n-2}(s, v) d s\right) d v,
\end{aligned}
$$

where $R_{0, n-2}$ is obtained by taking $c=0$ in (26).

Proof We give the proof of our results by fixing $j=1$, other cases can be followed in a similar pattern. By using (8) and (18) for $\psi$ and $\psi^{\prime}$ respectively and applying assumption $\psi(0)=0$, we get

$$
\mathbb{S}_{2}(\psi, f, d)=\int_{0}^{d} \mathbb{S}_{2}\left(G_{*, 1}(\cdot, s), f, d\right) \psi^{\prime \prime}(s) d s
$$

The rest is a similar application of (25) as in the proof of Theorem 4.

Similar to Theorem 5 (from Theorem 4), we may get the following theorem (from Theorem 7).

Theorem 8 Consider $\left(A_{2}\right)$ and let $f$ be as in Corollary $2(i)$. If $\psi$ is $n$-convex and

$$
\int_{0}^{d} \mathbb{S}_{2}\left(G_{*, j}(\cdot, s), f, d\right) \tilde{R}_{0, n-2}(s, v) d s \geq 0
$$

then

$$
\begin{aligned}
& \mathbb{S}_{2}(\psi, f, d) \\
& \geq \frac{\psi^{\prime}(0)-\psi^{\prime}(d)}{d} \int_{0}^{d} \mathbb{S}_{2}\left(G_{*, j}(\cdot, s), f, d\right) d s \\
& \quad+\frac{1}{d} \int_{0}^{d} \mathbb{S}_{2}\left(G_{*, j}(\cdot, s), f, d\right)\left(\sum_{l=2}^{n-1} \frac{l}{(l-1) !}\left(\psi^{(l)}(0)(s)^{l-1}-\psi^{(l)}(d)(s-d)^{l-1}\right)\right) d s
\end{aligned}
$$

and if

$$
\int_{0}^{d} \mathbb{S}_{2}\left(G_{*, j}(\cdot, s), f, d\right) R_{0, n-2}(s, v) d s \geq 0,
$$

then

$$
\begin{aligned}
\mathbb{S}_{2}(\psi, f, d) \geq & \left(\frac{\psi^{\prime}(d)-\psi^{\prime}(0)}{d}\right) \int_{0}^{d} \mathbb{S}_{2}\left(G_{*, j}(\cdot, s), f, d\right) d s \\
& +\frac{1}{d} \int_{0}^{d} \mathbb{S}_{2}\left(G_{*, j}(\cdot, s), f, d\right)\left(\sum_{l=3}^{n-1} \frac{f^{(l)}(0)(s)^{l-1}-f^{(l)}(d)(s-d)^{l-1}}{(l-3) !(l-1)}\right) d s
\end{aligned}
$$

for $j=1,2$, where $\tilde{R}_{0, n-2}$ and $R_{0, n-2}$ are as described in Theorem 7 .

The following theorem yields generalization of (6). 
Theorem 9 Consider $\left(A_{2}\right)$ and let $f$ be as in Corollary 2(i). If $\psi$ and

(i) If $n$ is even and $n \geq 4$, then (42) holds.

(ii) Let inequality (42) be satisfied and

$$
\sum_{l=1}^{n-1} \frac{l}{(l-1) !}\left(\psi^{(l)}(0)(s)^{l-1}-\psi^{(l)}(d)(s-d)^{l-1}\right) ; \quad \forall s \in[0, d]
$$

OR

(44) be satisfied and

$$
\psi^{\prime}(d)-\psi^{\prime}(0)+\sum_{l=3}^{n-1} \frac{\psi^{(l)}(0)(s)^{l-1}-\psi^{(l)}(d)(s-d)^{l-1}}{(l-3) !(l-1)} \geq 0 \quad \forall s \in[0, d],
$$

then we have

$$
\mathbb{S}_{2}(\psi, f, d) \geq 0
$$

Proof The proof is similar to that of Theorem 6 except for the use of Theorem 8 and Corollary 2(a).

Remark 3 Inequalities (42) and (44) hold in reverse directions if either the inequalities in (41) and (43) are reversed or $-\psi$ is $n$-convex. By using these reverse inequalities and applying a similar technique as in Theorem 9 , one may prove $\mathbb{S}_{2}(\psi, f, d) \leq 0$, which gives the reverse of (6).

For the generalization of (7), we construct the following identities.

Theorem 10 Consider $\left(A_{2}\right)$ and let $f$ be as in Corollary 2(i), then

(a)

$$
\begin{aligned}
\mathbb{S}_{3}(\psi, f, \lambda, d)= & \frac{\psi^{\prime}(0)-\psi^{\prime}(d)}{d} \int_{0}^{d} \mathbb{S}_{3}\left(G_{*, j}(\cdot, s), f, \lambda, d\right) d s \\
& +\frac{1}{d} \int_{0}^{d} \mathbb{S}_{3}\left(G_{*, j}(\cdot, s), f, \lambda, d\right) \\
& \times\left(\sum_{l=2}^{n-1} \frac{l}{(l-1) !}\left(\psi^{(l)}(0)(s)^{l-1}-\psi^{(l)}(d)(s-d)^{l-1}\right)\right) d s \\
& +\frac{1}{(n-3) !} \int_{0}^{d} \psi^{(n)}(v)\left(\int_{0}^{d} \mathbb{S}_{3}\left(G_{*, j}(\cdot, s), f, \lambda, d\right) \tilde{R}_{0, n-2}(s, v) d s\right) d v
\end{aligned}
$$

for $j=1,2$.

(b) If $\psi^{\prime}(0)=0$, then

$$
\begin{aligned}
\mathbb{S}_{3}(\psi, f, \lambda, d)+\psi(d) \int_{0}^{d} \lambda(x) d x \\
=\frac{\psi^{\prime}(0)-\psi^{\prime}(d)}{d} \int_{0}^{d} \mathbb{S}_{3}\left(G_{*, 3}(\cdot, s), f, \lambda, d\right) d s
\end{aligned}
$$




$$
\begin{aligned}
& +\frac{1}{d} \int_{0}^{d} \mathbb{S}_{3}\left(G_{*, 3}(\cdot, s), f, \lambda, d\right)\left(\sum_{l=2}^{n-1} \frac{l}{(l-1) !}\left(\psi^{(l)}(0)(s)^{l-1}-\psi^{(l)}(d)(s-d)^{l-1}\right)\right) \\
& +\frac{1}{(n-3) !} \int_{0}^{d} \psi^{(n)}(v)\left(\int_{0}^{d} \mathbb{S}_{3}\left(G_{*, 3}(\cdot, s), f, \lambda, d\right) \tilde{R}_{0, n-2}(s, v) d s\right) d v
\end{aligned}
$$

(c)

$$
\begin{aligned}
\mathbb{S}_{3}(\psi, f, \lambda, d)+\left(\psi(d)-d \psi^{\prime}(d)\right) \int_{0}^{d} \lambda(x) d x \\
=\frac{\psi^{\prime}(0)-\psi^{\prime}(d)}{d} \int_{0}^{d} \mathbb{S}_{3}\left(G_{*, 4}(\cdot, s), f, \lambda, d\right) d s \\
\quad+\frac{1}{d} \int_{0}^{d} \mathbb{S}_{3}\left(G_{*, 4}(\cdot, s), f, \lambda, d\right) \\
\quad \times\left(\sum_{l=2}^{n-1} \frac{l}{(l-1) !}\left(\psi^{(l)}(0)(s)^{l-1}-\psi^{(l)}(d)(s-d)^{l-1}\right)\right) d s \\
\quad+\frac{1}{(n-3) !} \int_{0}^{d} \psi^{(n)}(v)\left(\int_{0}^{d} \mathbb{S}_{3}\left(G_{*, 4}(\cdot, s), h, k, b\right) \tilde{R}_{0, n-2}(s, v) d s\right) d v .
\end{aligned}
$$

(d) If $\psi^{\prime}(0)=0$, then

$$
\begin{aligned}
\mathbb{S}_{3}(\psi, f, \lambda, d)-d \psi^{\prime}(d) \int_{0}^{d} \lambda(x) d x \\
=\frac{\psi^{\prime}(0)-\psi^{\prime}(d)}{d} \int_{0}^{d} \mathbb{S}_{3}\left(G_{*, 5}(\cdot, s), f, \lambda, d\right) d s \\
\quad+\frac{1}{d} \int_{0}^{d} \mathbb{S}_{3}\left(G_{*, 5}(\cdot, s), f, \lambda, d\right) \\
\quad \times\left(\sum_{l=2}^{n-1} \frac{l}{(l-1) !}\left(\psi^{(l)}(0)(s)^{l-1}-\psi^{(l)}(d)(s-d)^{l-1}\right)\right) d s \\
\quad+\frac{1}{(n-3) !} \int_{0}^{d} \psi^{(n)}(v)\left(\int_{0}^{d} \mathbb{S}_{3}\left(G_{*, 5}(\cdot, s), f, \lambda, d\right) \tilde{R}_{0, n-2}(s, v) d s\right) d v
\end{aligned}
$$

and

$\left(a^{\prime}\right)$

$$
\begin{aligned}
\mathbb{S}_{3}(\psi, f, \lambda, d) \\
=\left(\frac{\psi^{\prime}(d)-\psi^{\prime}(0)}{d}\right) \int_{0}^{d} \mathbb{S}_{3}\left(G_{*, j}(\cdot, s), f, \lambda, d\right) d s \\
\quad+\frac{1}{d} \int_{0}^{d} \mathbb{S}_{3}\left(G_{*, j}(\cdot, s), f, \lambda, d\right)\left(\sum_{l=3}^{n-1} \frac{\psi^{(l)}(0)(s)^{l-1}-\psi^{(l)}(d)(s-d)^{l-1}}{(l-3) !(l-1)}\right) d s \\
\quad+\frac{1}{(n-3) !} \int_{0}^{d} \psi^{(n)}(v)\left(\int_{0}^{d} \mathbb{S}_{3}\left(G_{*, j}(\cdot, s), f, \lambda, d\right) R_{0, n-2}(s, v) d s\right) d v
\end{aligned}
$$

for $j=1,2$. 
(b') If $\psi^{\prime}(0)=0$, then

$$
\begin{aligned}
\mathbb{S}_{3}(\psi, f, \lambda, d)+\psi(d) \int_{0}^{d} \lambda(x) d x \\
=\left(\frac{\psi^{\prime}(d)-\psi^{\prime}(0)}{d}\right) \int_{0}^{d} \mathbb{S}_{3}\left(G_{*, 3}(\cdot, s), f, \lambda, d\right) d s \\
\quad+\frac{1}{d} \int_{0}^{d} \mathbb{S}_{3}\left(G_{*, 3}(\cdot, s), f, \lambda, d\right)\left(\sum_{l=3}^{n-1} \frac{\psi^{(l)}(0)(s)^{l-1}-\psi^{(l)}(d)(s-d)^{l-1}}{(l-3) !(l-1)}\right) d s \\
\quad+\frac{1}{(n-3) !} \int_{0}^{d} \psi^{(n)}(v)\left(\int_{0}^{d} \mathbb{S}_{3}\left(G_{*, 3}(\cdot, s), f, \lambda, d\right) R_{0, n-2}(s, v) d s\right) d v .
\end{aligned}
$$

$\left(c^{\prime}\right)$

$$
\begin{aligned}
\mathbb{S}_{3}(\psi, f, \lambda, d)+\left(\psi(d)-d \psi^{\prime}(d)\right) \int_{0}^{d} \lambda(x) d x \\
=\left(\frac{\psi^{\prime}(d)-\psi^{\prime}(0)}{d}\right) \int_{0}^{d} \mathbb{S}_{3}\left(G_{*, 4}(\cdot, s), f, \lambda, d\right) d s \\
\quad+\frac{1}{d} \int_{0}^{d} \mathbb{S}_{3}\left(G_{*, 4}(\cdot, s), f, \lambda, d\right)\left(\sum_{l=3}^{n-1} \frac{\psi^{(l)}(0)(s)^{l-1}-\psi^{(l)}(d)(s-d)^{l-1}}{(l-3) !(l-1)}\right) d s \\
\quad+\frac{1}{(n-3) !} \int_{0}^{d} \psi^{(n)}(v)\left(\int_{0}^{d} \mathbb{S}_{3}\left(G_{*, 4}(\cdot, s), f, \lambda, d\right) R_{0, n-2}(s, v) d s\right) d v .
\end{aligned}
$$

(d') If $\psi^{\prime}(0)=0$, then

$$
\begin{aligned}
\mathbb{S}_{3}(\psi, f, \lambda, d)-d \psi^{\prime}(d) \int_{0}^{d} \lambda(x) d x \\
=\left(\frac{\psi^{\prime}(d)-\psi^{\prime}(0)}{d}\right) \int_{0}^{d} \mathbb{S}_{3}\left(G_{*, 5}(\cdot, s), f, \lambda, d\right) d s \\
\quad+\frac{1}{d} \int_{0}^{d} \mathbb{S}_{3}\left(G_{*, 5}(\cdot, s), f, \lambda, d\right)\left(\sum_{l=3}^{n-1} \frac{\psi^{(l)}(0)(s)^{l-1}-\psi^{(l)}(d)(s-d)^{l-1}}{(l-3) !(l-1)}\right) d s \\
\quad+\frac{1}{(n-3) !} \int_{0}^{d} \psi^{(n)}(v)\left(\int_{0}^{d} \mathbb{S}_{3}\left(G_{*, 5}(\cdot, s), f, \lambda, d\right) R_{0, n-2}(s, v) d s\right) d v,
\end{aligned}
$$

where $\tilde{R}_{0, n-2}$ and $R_{0, n-2}$ are as described in Theorem 7 .

Proof We give the proof of our results by fixing $j=1$, other cases can be followed in a similar pattern. By using (8) and (18) for $\psi$ and $\psi^{\prime}, \psi(0)=0$ and $\int_{0}^{d} \lambda(x) \int_{0}^{x} f(t) d t d x=$ $\int_{0}^{d} f(t)\left(\int_{t}^{b} \lambda(x) d x\right) d t=\int_{0}^{d} \Lambda(t) f(t) d t$, we get

$$
\mathbb{S}_{3}(\psi, f, \lambda, d)=\int_{0}^{d} \mathbb{S}_{3}\left(G_{*, 1}(\cdot, s), f, \lambda, d\right) \psi^{\prime \prime}(s) d s .
$$

The rest is a similar application of (25).

Similar to Theorem 5 (from Theorem 4), we may get the following theorem (from Theorem 10). 
Theorem 11 Consider $\left(A_{2}\right)$ and let $f$ be as in Corollary 2(i). If $\psi$ is $n$-convex and

$$
\int_{0}^{d} \mathbb{S}_{3}\left(G_{*, j}(\cdot, s), f, \lambda, d\right) \tilde{R}_{0, n-2}(s, v) d s \geq 0
$$

then

$$
\begin{aligned}
\mathbb{S}_{3}(\psi, f, \lambda, d) \geq & \frac{\psi^{\prime}(0)-\psi^{\prime}(d)}{d} \int_{0}^{d} \mathbb{S}_{3}\left(G_{*, j}(\cdot, s), f, \lambda, d\right) d s \\
& +\frac{1}{d} \int_{0}^{d} \mathbb{S}_{3}\left(G_{*, j}(\cdot, s), f, \lambda, d\right) \\
& \times\left(\sum_{l=2}^{n-1} \frac{l}{(l-1) !}\left(\psi^{(l)}(0)(s)^{l-1}-\psi^{(l)}(d)(s-d)^{l-1}\right)\right) d s ;
\end{aligned}
$$

and if

$$
\int_{0}^{d} \mathbb{S}_{3}\left(G_{*, j}(\cdot, s), f, \lambda, d\right) R_{0, n-2}(s, v) d s \geq 0
$$

then

$$
\begin{aligned}
\mathbb{S}_{3}(\psi, f, \lambda, d) \geq & \left(\frac{\psi^{\prime}(d)-\psi^{\prime}(0)}{d}\right) \int_{0}^{d} \mathbb{S}_{3}\left(G_{*, j}(\cdot, s), f, \lambda, d\right) d s \\
& +\frac{1}{d} \int_{0}^{d} \mathbb{S}_{3}\left(G_{*, j}(\cdot, s), f, \lambda, d\right) \\
& \times\left(\sum_{l=3}^{n-1} \frac{\psi^{(l)}(0)(s)^{l-1}-\psi^{(l)}(d)(s-d)^{l-1}}{(l-3) !(l-1)}\right) d s
\end{aligned}
$$

for $j=1,2$, where $\tilde{R}_{0, n-2}$ and $R_{0, n-2}$ are as described in Theorem 7 .

In the next theorem, we prove generalization of (7).

Theorem 12 Consider $\left(A_{2}\right)$ and let $f, \lambda, \Lambda$ be as in Corollary 3(i). If $\psi$ is $n$-convex and

(i) If $n$ is even and $n \geq 4$, then (49) holds.

(ii) Let inequality (49) be satisfied and

$$
\sum_{l=1}^{n-1} \frac{l}{(l-1) !}\left(\psi^{(l)}(0)(s)^{l-1}-\psi^{(l)}(d)(s-d)^{l-1}\right) ; \quad \forall s \in[0, d]
$$

OR

(51) be satisfied and

$$
\psi^{\prime}(d)-\psi^{\prime}(0)+\sum_{l=3}^{n-1} \frac{\psi^{(l)}(0)(s)^{l-1}-\psi^{(l)}(d)(s-d)^{l-1}}{(l-3) !(l-1)} \geq 0 ; \quad \forall s \in[0, d] .
$$

Then we have

$$
\mathbb{S}_{3}(\psi, f, \lambda, d) \geq 0 \text {. }
$$


Proof The proof is an application of Theorem 10, Theorem 11, and Corollary 3(a).

Remark 4 Inequalities (49) and (51) hold in reverse directions if either the inequalities in (48) and (50) are reversed or $-\psi$ is $n$-convex. By using these reverse inequalities and applying similar technique as in Theorem 12 , one may prove $\mathbb{S}_{3}(\psi, f, \lambda, d) \leq 0$, which gives the reverse of (7).

Remark 5 Theorem 8 and Theorem 11 have been proved for $j=1,2$. Both theorems can be extended to $j=1,2, \ldots, 5$ according to each case in Theorem 7 and Theorem 10, respectively, which ultimately produce inequalities (as given in Theorem 9 and Theorem 12) related to the generalizations of (6) and (7) (and their reverse).

\section{New upper bounds via Čebyšev functional}

Consider the Čebyšev functional for two Lebesgue integrable functions $\mathbb{F}_{1}, \mathbb{F}_{2}:[c, d] \rightarrow \mathbb{R}$ given as follows:

$$
T\left(\mathbb{F}_{1}, \mathbb{F}_{2}\right)=\frac{1}{d-c} \int_{c}^{d} \mathbb{F}_{1}(\xi) \mathbb{F}_{2}(\xi) d \xi-\frac{1}{d-c} \int_{c}^{d} \mathbb{F}_{1}(\xi) d \xi \frac{1}{d-c} \int_{c}^{d} \mathbb{F}_{2}(\xi) d \xi
$$

Dragomir et al. in [3] proposed new bounds utilizing the Čebyšev functional given as follows.

Theorem 13 For $\mathbb{F}_{1} \in L[c, d]$ and $\mathbb{F}_{2}:[c, d] \rightarrow \mathbb{R}$ being absolutely-continuous functions along with $(\cdot-c)(d-\cdot)\left[\mathbb{F}_{2}^{\prime}\right]^{2} \in L[c, d]$, the following inequality holds:

$$
\left|T\left(\mathbb{F}_{1}, \mathbb{F}_{2}\right)\right| \leq \frac{1}{\sqrt{2}}\left[\frac{T\left(\mathbb{F}_{1}, \mathbb{F}_{1}\right)}{(d-c)}\right]^{\frac{1}{2}}\left(\int_{c}^{d}(\xi-c)(d-\xi)\left[\mathbb{F}_{2}^{\prime}(\xi)\right]^{2} d \xi\right)^{\frac{1}{2}}
$$

Theorem 14 For $\mathbb{F}_{1}:[c, d] \rightarrow \mathbb{R}$ being absolutely continuous with $\mathbb{F}_{1}^{\prime} \in L_{\infty}[c, d]$ and $\mathbb{F}_{2}$ : $[c, d] \rightarrow \mathbb{R}$ being an increasing function, the following inequality holds:

$$
\left|T\left(\mathbb{F}_{1}, \mathbb{F}_{2}\right)\right| \leq \frac{\left\|\mathbb{F}_{1}^{\prime}\right\|_{\infty}}{2(d-c)} \int_{c}^{d}(\xi-c)(d-\xi) d \mathbb{F}_{2}(\xi) .
$$

The constants $\frac{1}{\sqrt{2}}$ and $\frac{1}{2}$ are the optimal constants.

Now we utilize above theorems to construct new upper bounds for our obtained generalized identities. For our convenience, we note

$$
\tilde{\mathfrak{S}}_{j}(v)=\int_{c}^{d} \mathbb{S}_{1}\left(G_{*, j}(\cdot, s), f, c, d\right) \tilde{R}_{n-2}(s, v) d s, \quad v \in[c, d]
$$

and

$$
\mathfrak{S}_{j}(v)=\int_{c}^{d} \mathbb{S}_{1}\left(G_{*, j}(\cdot, s), f, c, d\right) R_{n-2}(s, v) d s, \quad v \in[c, d]
$$

for $\{j=1, \ldots, 5\}$. Consider the Čebyšev functionals $T_{j}\left(\tilde{\mathfrak{S}}_{j}, \tilde{\mathfrak{S}}_{\mathfrak{j}}\right)$ and $T_{j}\left(\mathfrak{S}_{\mathfrak{j}}, \mathfrak{S}_{\mathbf{j}}\right)\{j=1, \ldots, 5\}$ given as

$$
T_{j}\left(\tilde{\mathfrak{S}}_{j}, \tilde{\mathfrak{S}}_{j}\right)=\frac{1}{d-c} \int_{c}^{d} \tilde{\mathfrak{S}}_{\mathbf{j}}^{2}(\xi) d \xi-\left(\frac{1}{d-c} \int_{c}^{d} \tilde{\mathfrak{S}}_{\mathbf{j}}(\xi) d \xi\right)^{2}
$$


and

$$
T_{j}\left(\mathfrak{S}_{\mathrm{j}}, \mathfrak{S}_{\mathrm{j}}\right)=\frac{1}{d-c} \int_{c}^{d} \mathfrak{S}_{\mathrm{j}}{ }^{2}(\xi) d \xi-\left(\frac{1}{d-c} \int_{c}^{d} \mathfrak{S}_{\mathrm{j}}(\xi) d \xi\right)^{2}
$$

respectively.

Grüss type inequalities associated with Theorem 13 and Theorem 14 can be given as follows.

Theorem 15 Under the assumptions of Theorem 4 , let $\psi:[c, d] \rightarrow \mathbb{R}$ be absolutely continuous along with $(\cdot-c)(d-\cdot)\left[\psi^{(n+1)}\right]^{2} \in L[c, d]$ and $\tilde{\mathfrak{S}}_{\mathrm{j}}, \mathfrak{S}_{\mathrm{j}} j \in\{1,2, \ldots, 5\}$ be defined in (57), (58) respectively. Then

$$
\begin{aligned}
\mathbb{S}_{1}(\psi, f, c, d)-\frac{\psi^{\prime}(c)-\psi^{\prime}(d)}{d-c} \int_{c}^{d} \mathbb{S}_{1}\left(G_{*, j}(\cdot, s), f, c, d\right) d s \\
-\frac{1}{d-c} \int_{c}^{d} \mathbb{S}_{1}\left(G_{*, j}(\cdot, s), f, c, d\right) \\
\quad \times\left(\sum_{l=2}^{n-1} \frac{l}{(l-1) !}\left(\psi^{(l)}(c)(s-c)^{l-1}-\psi^{(l)}(d)(s-d)^{l-1}\right)\right) d s \\
-\frac{\psi^{(n-1)}(d)-\psi^{(n-1)}(c)}{(d-c)(n-3) !} \int_{c}^{d} \tilde{\mathfrak{S}}_{\mathbf{j}}(v) d v=\operatorname{Rem}\left(c, d, \tilde{\mathfrak{S}}_{j}, \psi^{(n)}\right)
\end{aligned}
$$

and

$$
\begin{aligned}
& \mathbb{S}_{1}(\psi, f, c, d)-\left(\frac{\psi^{\prime}(d)-\psi^{\prime}(c)}{d-c}\right) \int_{c}^{d} \mathbb{S}_{1}\left(G_{*, j}(\cdot, s), f, c, d\right) d s \\
& -\frac{1}{d-c} \int_{c}^{d} \mathbb{S}_{1}\left(G_{*, j}(\cdot, s), f, c, d\right)\left(\sum_{l=3}^{n-1} \frac{\psi^{(l)}(c)(s-c)^{l-1}-\psi^{(l)}(d)(s-d)^{l-1}}{(l-3) !(l-1)}\right) d s \\
& -\frac{\psi^{(n-1)}(d)-\psi^{(n-1)}(c)}{(d-c)(n-3) !} \int_{c}^{d} \mathfrak{S}_{\mathrm{j}}(v) d v=\operatorname{Rem}\left(c, d, \mathfrak{S}_{\mathrm{j}}, \psi^{(n)}\right),
\end{aligned}
$$

where

$$
\left|\operatorname{Rem}\left(c, d, \tilde{\mathfrak{S}}_{\mathrm{j}}, \psi^{(n)}\right)\right| \leq \frac{\left[T_{j}\left(\tilde{\mathfrak{S}}_{\mathrm{j}}, \tilde{\mathfrak{S}}_{\mathrm{j}}\right)\right]^{\frac{1}{2}}}{(n-3) !} \sqrt{\frac{d-c}{2}}\left|\int_{c}^{d}(v-c)(d-v)\left[\psi^{(n+1)}(v)\right]^{2} d v\right|^{\frac{1}{2}}
$$

and

$$
\left|\operatorname{Rem}\left(c, d, \mathfrak{S}_{\mathrm{j}}, \psi^{(n)}\right)\right| \leq \frac{\left[T_{j}\left(\mathfrak{S}_{\mathrm{j}}, \mathfrak{S}_{\mathrm{j}}\right)\right]^{\frac{1}{2}}}{(n-3) !} \sqrt{\frac{d-c}{2}}\left|\int_{c}^{d}(v-c)(d-v)\left[\psi^{(n+1)}(v)\right]^{2} d \nu\right|^{\frac{1}{2}}
$$

Proof Fix $j \in\{1,2, \ldots, 5\}$. Using the Čebyšev functional for $\mathbb{F}_{1}=\tilde{\mathfrak{S}}_{\mathrm{j}}, \mathbb{F}_{2}=\psi^{(n)}$ and by comparing (61) with (27), we have

$$
\operatorname{Rem}\left(c, d, \mathfrak{S}_{\mathbf{j}}, \psi^{(n)}\right)=\frac{d-c}{(n-3) !} T_{j}\left(\mathfrak{S}_{\mathrm{j}}, f^{(n)}\right)
$$

Employing Theorem 13 for the new functions, we get the required bound. 
Similarly, we can construct the other bound by using the Čebyšev functional for $\mathbb{F}_{1}=\mathfrak{S}_{j}$, $\mathbb{F}_{2}=\psi^{(n)}$ and by comparing (62) with (30).

Theorem 16 Under the assumptions of Theorem 4 , let $\psi:[c, d] \rightarrow \mathbb{R}$ be absolutely continuous alongwith $\psi^{(n+1)} \geq 0$ and $\tilde{\mathfrak{S}}_{j}, \mathfrak{S}_{j}\{j=1,2,3,4,5\}$ be defined in (57), (58) respectively. Then $\operatorname{Rem}\left(c, d, \tilde{\mathfrak{S}}_{\mathfrak{j}}, \psi^{(n)}\right)$ in $(61)$ and $\operatorname{Rem}\left(c, d, \mathfrak{S}_{\mathfrak{j}}, \psi^{(n)}\right)$ in $(62)$ satisfy bounds

$$
\begin{aligned}
& \left|\operatorname{Rem}\left(c, d, \tilde{\mathfrak{S}}_{j}, \psi^{(n)}\right)\right| \\
& \quad \leq \frac{(d-c)\left\|\tilde{\mathfrak{S}}_{j}^{\prime}\right\|_{\infty}}{(n-3) !}\left[\frac{\psi^{(n-1)}(d)+\psi^{(n-1)}(c)}{2}-\frac{\psi^{(n-2)}(d)-\psi^{(n-2)}(c)}{d-c}\right]
\end{aligned}
$$

and

$$
\begin{aligned}
& \left|\operatorname{Rem}\left(c, d, \mathfrak{S}_{\mathrm{j}}, \psi^{(n)}\right)\right| \\
& \quad \leq \frac{(d-c)\left\|\mathfrak{S}_{\mathbf{j}}^{\prime}\right\|_{\infty}}{(n-3) !}\left[\frac{\psi^{(n-1)}(d)+\psi^{(n-1)}(c)}{2}-\frac{\psi^{(n-2)}(d)-\psi^{(n-2)}(c)}{d-c}\right]
\end{aligned}
$$

respectively.

Proof We gave proof for one bound (63), and that for the other bound (64) can be obtained similarly. Since we have established

$$
\operatorname{Rem}\left(c, d, \tilde{\mathfrak{S}}_{j}, \psi^{(n)}\right)=\frac{d-c}{(n-3) !} T_{j}\left(\tilde{\mathfrak{S}}_{j}, \psi^{(n)}\right)
$$

now, applying Theorem 14 for $\mathbb{F}_{1}=\tilde{\mathfrak{S}}_{\mathbf{j}}, \mathbb{F}_{2}=\psi^{(n)}$, we have

$$
\begin{aligned}
\left|\operatorname{Rem}\left(c, d, \tilde{\mathfrak{S}}_{j}, \psi^{(n)}\right)\right| & =\frac{d-c}{(n-3) !}\left|T_{j}\left(\tilde{\mathfrak{S}}_{j}, \psi^{(n)}\right)\right| \\
& \leq \frac{\left\|\mathfrak{S}_{j}^{\prime}\right\|_{\infty}}{2(n-3) !} \int_{c}^{d}(v-c)(d-v) \psi^{(n+1)}(v) d v
\end{aligned}
$$

Now since

$$
\begin{aligned}
\int_{c}^{d}(v-c)(d-v) \psi^{(n+1)}(v) d v & =\int_{c}^{d}[2 v-(c+d)] \psi^{(n)}(v) d v \\
& =(d-c)\left[\psi^{(n-1)}(d)+\psi^{(n-1)}(c)\right]-2\left(\psi^{(n-2)}(d)-f^{(n-2)}(c)\right),
\end{aligned}
$$

therefore the required bound (63) follows.

Ostrowski type inequalities associated with generalized Steffensen's inequality can be given as follows.

Theorem 17 Under the assumptions of Theorem 4 , let $\left|\psi^{(n)}\right|^{p}:[c, d] \rightarrow \mathbb{R}$ be an $R$ integrable function, and consider $\left(p, p^{\prime}\right)$ to be a pair of conjugate exponents from $[1, \infty]$ 
such that $\frac{1}{p}+\frac{1}{p^{\prime}}=1$. Then we have

$$
\begin{aligned}
& \mid \mathbb{S}_{1}(\psi, f, c, d)-\frac{\psi^{\prime}(c)-\psi^{\prime}(d)}{d-c} \int_{c}^{d} \mathbb{S}_{1}\left(G_{*, j}(\cdot, s), f, c, d\right) d s \\
& \quad-\frac{1}{d-c} \int_{c}^{d} \mathbb{S}_{1}\left(G_{*, j}(\cdot, s), f, c, d\right) \\
& \quad \times\left(\sum_{l=2}^{n-1} \frac{l}{(l-1) !}\left(\psi^{(l)}(c)(s-c)^{l-1}-\psi^{(l)}(d)(s-d)^{l-1}\right)\right) d s \mid \\
& \leq \frac{\left\|\psi^{(n)}\right\|_{p}}{(n-3) !}\left(\int_{c}^{d}\left|\mathbb{S}_{1}\left(G_{*, j}(\cdot, s), f, c, d\right) \tilde{R}_{n-2}(s, v) d s\right|^{p^{\prime}} d v\right)^{1 / p^{\prime}}
\end{aligned}
$$

and

$$
\begin{aligned}
& \mid \mathbb{S}_{1}(\psi, f, c, d)-\left(\frac{\psi^{\prime}(d)-\psi^{\prime}(c)}{d-c}\right) \int_{c}^{d} \mathbb{S}_{1}\left(G_{*, j}(\cdot, s), f, c, d\right) d s \\
& \quad-\frac{1}{d-c} \int_{c}^{d} \mathbb{S}_{1}\left(G_{*, j}(\cdot, s), f, c, d\right)\left(\sum_{l=3}^{n-1} \frac{\psi^{(l)}(c)(s-c)^{l-1}-\psi^{(l)}(d)(s-d)^{l-1}}{(l-3) !(l-1)}\right) d s \mid \\
& \leq \frac{\left\|\psi^{(n)}\right\|_{p}}{(n-3) !}\left(\int_{c}^{d}\left|\mathbb{S}_{1}\left(G_{*, j}(\cdot, s), f, c, d\right) R_{n-2}(s, v) d s\right|^{p^{\prime}} d v\right)^{1 / p^{\prime}}
\end{aligned}
$$

respectively. The constant on the R.H.S. of (65) and (66) is sharp for $1<p \leq \infty$ and the best possible for $p=1$.

Proof Fix $j \in\{1,2, \ldots, 5\}$. Let us denote

$$
\tilde{\mathfrak{I}}_{\mathfrak{j}}=\frac{1}{(n-3) !}\left(\mathbb{S}_{1}\left(G_{*, j}(\cdot, s), f, c, d\right) \tilde{R}_{n-2}(s, v) d s\right), \quad v \in[c, d] .
$$

Using identity (27), we find

$$
\begin{aligned}
& \mid \mathbb{S}_{1}(\psi, f, c, d)-\frac{\psi^{\prime}(c)-\psi^{\prime}(d)}{d-c} \int_{c}^{d} \mathbb{S}_{1}\left(G_{*, j}(\cdot, s), f, c, d\right) d s \\
& \quad-\frac{1}{d-c} \int_{c}^{d} \mathbb{S}_{1}\left(G_{*, j}(\cdot, s), f, c, d\right) \\
& \quad \times\left(\sum_{l=2}^{n-1} \frac{l}{(l-1) !}\left(\psi^{(l)}(c)(s-c)^{l-1}-\psi^{(l)}(d)(s-d)^{l-1}\right)\right) d s \mid \\
& =\left|\int_{c}^{d} \tilde{\mathfrak{I}}_{j}(t) \psi^{(n)}(v) d v\right| .
\end{aligned}
$$

Applying Hölder's inequality for integrals on the R.H.S. of (67), we obtain

$$
\left|\int_{c}^{d} \tilde{\mathfrak{I}}_{\mathfrak{j}}(v) \psi^{(n)}(v) d v\right| \leq\left(\int_{c}^{d}\left|\psi^{(n)}(v)\right|^{p} d v\right)^{\frac{1}{p}}\left(\int_{c}^{d}\left|\tilde{\mathfrak{I}}_{\mathfrak{j}}(v)\right|^{p^{\prime}} d v\right)^{\frac{1}{p^{\prime}}}
$$

which combined together with (67) gives (65). 
For sharpness of the constant $\left(\int_{c}^{d}\left|\tilde{\mathfrak{I}}_{\mathfrak{j}}(v)\right|^{p^{\prime}} d v\right)^{1 / p^{\prime}}$, see [2].

Similarly, we can prove (66).

Remark 6 Similar bounds of Grüss and Ostrowski type inequalities can be obtained by using Theorem 7 and Theorem 10.

\section{Monotonic Steffensen-type functionals}

The notion of $(n+1)$-convex function at a point was introduced in [15]. In the current section, we define some linear functionals from the differences of generalized Steffensentype inequalities. By proving monotonicity of these functionals, we obtain new inequalities which contribute to theory of more generalized class of functions, i.e., $(n+1)$-convex functions at a point. The following is the definition of $(n+1)$-convex function at a point, see [15].

Definition 1 Let $I \subseteq \mathbb{R}$ be an interval, $\xi \in I^{0}$, and $n \in \mathbb{N}$. A function $f: I \rightarrow \mathbb{R}$ is said to be $(n+1)$-convex at point $\xi$ if there exists a constant $K_{\xi}$ such that the function

$$
F(x)=f(x)-K_{\xi} \frac{x^{n}}{n !}
$$

is $n$-concave on $I \cap(-\infty, \xi]$ and $n$-convex on $I \cap[\xi, \infty)$.

Pečarić et al. in [15] studied necessary and sufficient conditions on two linear functionals $\Omega: C\left(\left[\delta_{1}, \xi\right]\right) \rightarrow \mathbb{R}$ and $\Gamma: C\left(\left[\xi, \delta_{2}\right]\right) \rightarrow \mathbb{R}$ so that the inequality $\Omega(f) \leq \Gamma(f)$ holds for every function $f$ that is $(n+1)$-convex at point $\xi$. In this section, we define linear functionals and obtain such inequalities for defined functionals. Let $n \in \mathbb{N}, n \geq 2$ be even, $\psi:[c, d] \rightarrow \mathbb{R}$ be an $n$ times differentiable function with $\psi^{(n-1)}$ absolutely continuous on $[c, d]$. Let $c_{1}, c_{2} \in[c, d]$ and $\xi \in(c, d)$, where $c_{1}<\xi<c_{2}$. Let $f_{1}:\left[c_{1}, \xi\right] \rightarrow \mathbb{R}$ and $f_{2}:\left[\xi, c_{2}\right] \rightarrow \mathbb{R}$ be increasing with $f_{i}(t) \leq t$ for $i=1,2$. For $j=1,2, \ldots, 5$, we construct:

$$
\begin{aligned}
\Omega_{1, j}(\psi)= & \mathbb{S}_{1}\left(\psi, f_{1}, c_{1}, \xi\right)-\frac{\psi^{\prime}\left(c_{1}\right)-\psi^{\prime}(\xi)}{\xi-c_{1}} \int_{c_{1}}^{\xi} \mathbb{S}_{1}\left(G_{*, j}(\cdot, s), f_{1}, c_{1}, \xi\right) d s \\
& -\frac{1}{\xi-c_{1}} \int_{c_{1}}^{\xi} \mathbb{S}_{1}\left(G_{*, j}(\cdot, s), f_{1}, c_{1}, \xi\right) \\
& \times\left(\sum_{l=2}^{n-1} \frac{l}{(l-1) !}\left(\psi^{(l)}\left(c_{1}\right)\left(s-c_{1}\right)^{l-1}-\psi^{(l)}(\xi)(s-\xi)^{l-1}\right)\right) d s
\end{aligned}
$$

and

$$
\begin{aligned}
\Gamma_{1, j}(\psi)= & \mathbb{S}_{1}\left(\psi, f_{2}, \xi, c_{2}\right)-\frac{\psi^{\prime}(\xi)-\psi^{\prime}\left(c_{2}\right)}{c_{2}-\xi} \int_{\xi}^{c_{2}} \mathbb{S}_{1}\left(G_{*, j}(\cdot, s), f_{2}, \xi, c_{2}\right) d s \\
& -\frac{1}{c_{2}-\xi} \int_{\xi}^{c_{2}} \mathbb{S}_{1}\left(G_{*, j}(\cdot, s), f_{2}, \xi, c_{2}\right) \\
& \times\left(\sum_{l=2}^{n-1} \frac{l}{(l-1) !}\left(\psi^{(l)}(\xi)(s-\xi)^{l-1}-\psi^{(l)}\left(c_{2}\right)\left(s-c_{2}\right)^{l-1}\right)\right) d s .
\end{aligned}
$$


Theorem 5(a) enables $\Gamma_{1, j}(\psi) \geq 0$ for $j=1,2, \ldots, 5$ (and $\psi^{\prime}(0)=0$ for $j=3$ ) provided that $\psi$ is $n$-convex. Furthermore, Remark 2 enables $\Omega_{1, j}(\psi) \leq 0$ for $j=1,2, \ldots, 5$ (and $f^{\prime}(0)=0$ for $j=3$ ) provided that $-\psi$ is $n$-convex.

Theorem 18 Let $\psi, f_{1}, f_{2}$ be as defined above and $\psi:[c, d] \rightarrow \mathbb{R}$ be $(n+1)$-convex at a point $\xi$ for even $n>3$. If $\Omega_{1, j}\left(P_{n}\right)=\Gamma_{1, j}\left(P_{n}\right)$ for all $j=1,2, \ldots, 5$ and $\psi^{\prime}(0)=0$ for $j=3$, where $P_{n}(u)=u^{n}$, then

$$
\Omega_{1, j}(\psi) \leq \Gamma_{1, j}(\psi)
$$

for $j=1,2, \ldots, 5$.

Proof As $\psi$ is $(n+1)$-convex at $\xi$, so by Definition 1 there is $\exists K_{\xi}$ such that $\Psi(u)=\psi(u)-$ $\frac{K_{\xi} u^{n}}{n !}$ is $n$-concave on $\left[c_{1}, \xi\right]$ and $n$-convex on $\left[\xi, c_{2}\right]$. Therefore, for each $j=1,2, \ldots, 5$, we have

$$
\Omega_{1, j}(\psi)-\frac{K_{\xi}}{n !} \Omega_{1, j}\left(P_{n}\right)=\Omega_{1, j}(\Psi) \leq 0 \leq \Gamma_{1, j}(\Psi)=\Gamma_{1, j}(\psi)-\frac{K_{\xi}}{n !} \Gamma_{1, j}\left(P_{n}\right) .
$$

Since $\Omega_{1, j}\left(P_{n}\right)=\Gamma_{1, j}\left(P_{n}\right)$, therefore $\Omega_{1, j}(\psi) \leq \Gamma_{1, j}(\psi)$, which completes the proof.

Remark 7 We may proceed further by defining linear functionals with the inequalities proved in (37), Theorem 8 , and Theorem 11 . Moreover, by proving monotonicity of new functionals, we extend the inequalities from Theorem 5 , Theorem 8 , and Theorem 11.

\section{Application to exponentially convex functions}

We start this section by an important remark given as follows.

Remark 8 By the virtue of Theorem 5 , for $j=1,2, \ldots, 5$, we define the positive linear functionals with respect to $n$-convex function $\psi$ as follows:

$$
\begin{aligned}
\Delta_{1, j}(\psi):= & \mathbb{S}_{1}(\psi, f, c, d)-\frac{\psi^{\prime}(c)-\psi^{\prime}(d)}{d-c} \int_{c}^{d} \mathbb{S}_{1}\left(G_{*, j}(\cdot, s), f, c, d\right) d s \\
& -\frac{1}{d-c} \int_{c}^{d} \mathbb{S}_{1}\left(G_{*, j}(\cdot, s), f, c, d\right) \\
& \times\left(\sum_{l=2}^{n-1} \frac{l}{(l-1) !}\left(\psi^{(l)}(c)(s-c)^{l-1}-\psi^{(l)}(d)(s-d)^{l-1}\right)\right) d s \\
\geq & 0 .
\end{aligned}
$$

Next we construct the non-trivial examples of exponentially convex functions (see [8]) from positive linear functionals $\Delta_{1, j}(\psi)$ for $(j=1,2, \ldots, 5)$.

For this, consider a family of real-valued functions on $[0, \infty)$ given as

$$
\psi_{s}(u)= \begin{cases}\frac{u^{s}}{s(s-1) \cdots(s-n+1)}, & s \notin\{0,1, \ldots, n-1\} ; \\ \frac{u^{t} \ln u}{(-1)^{n-1-t} t !(n-1-t) !}, & s=t \in\{0,1, \ldots, n-1\} .\end{cases}
$$


It is interesting to note that this is a family of $n$-convex functions as

$$
\frac{d^{n}}{d u^{n}} \psi_{s}(u)=u^{s-n} \geq 0
$$

Since $s \mapsto u^{s-n}=e^{(s-n) \ln u}$ is an exponentially convex function, therefore the mapping $s \mapsto \Delta_{1, j}\left(\psi_{s}\right)$ is exponentially convex and, as a special case, it is also log-convex mapping. The log-convexity of this mapping enables us to construct the known Lyapunov inequality given as

$$
\left(\Delta_{1, j}\left(\psi_{s}\right)\right)^{t-r} \leq\left(\Delta_{1, j}\left(\psi_{r}\right)\right)^{t-s}\left(\Delta_{1, j}\left(\psi_{t}\right)\right)^{s-r}
$$

for $r, s, t \in \mathbb{R}$ such that $r<s<t$, where $j=1,2, \ldots, 5$.

Remark 9 We have not given the proof of the above mentioned results in detail (see [8]). Lyapunov inequality empowered us to refine lower (upper) bound for action of the functional on the class of functions given in (71) because if an exponentially convex mapping attains zero value at some point, it is zero everywhere (see [8]).

One can also consider some other classes of $n$-convex functions given in the paper [8] and can get similar estimations. A similar technique can also be employed by considering the results of (37), Theorem 8 , and Theorem 11.

\section{Acknowledgements}

All the authors are thankful to their respective institutes for excellent research facilities. The research of fourth author was supported by the Ministry of Education and Science of the Russian Federation (the Agreement number No. 02.a03.21.0008).

\section{Funding}

The research of the first and third authors was supported by the Higher Education Commission (H.E.C.) of Pakistan under NRPU project 5327.

\section{Competing interests}

The authors declare that they have no competing interests.

\section{Authors' contributions}

All authors jointly worked on the results and they read and approved the final manuscript.

\section{Author details}

${ }^{1}$ Department of Mathematics, COMSATS University Islamabad, Lahore Campus, Pakistan. ${ }^{2}$ Department of Mathematics, COMSATS University Islamabad, Vehari Campus, Pakistan. ${ }^{3}$ RUDN University, Moscow, Russia.

\section{Publisher's Note}

Springer Nature remains neutral with regard to jurisdictional claims in published maps and institutional affiliations.

Received: 25 March 2019 Accepted: 1 July 2019 Published online: 17 July 2019

\section{References}

1. Aljinović, A.A., Pečarić, J., Vukelić, A.: On some Ostrowski type inequalities via Montgomery identity and Taylor's formula II. Tamkang J. Math. 36(4), 279-301 (2005)

2. Butt, S.I., Khan, K.A., Pečarić, J.: Popoviciu type inequalities via green function and generalized Montgomery identity. Math. Inequal. Appl. 18(4), 1519-1538 (2015)

3. Cerone, P., Dragomir, S.S.: Some new Ostrowski-type bounds for the Čebyšev functional and applications. J. Math. Inequal. 8(1), 159-170 (2014)

4. Fahad, A., Pečarić, J., Praljak, M.: Generalized Steffensen's inequality. J. Math. Inequal. 9(2), 481-487 (2015)

5. Fahad, A., Pečarić, J., Qureshi, M.I.: Generalized Steffensen's inequality by Lidstone interpolation and Montogomery's identity. J. Inequal. Appl. 2018, 237 (2018)

6. Hewitt, E., Stromberg, K.: Real and Abstract Analysis, 3rd edn. Springer, New York (1975)

7. Iqbal, S., Pečarić, J., Samraiz, M.: Hardy-type inequalities in quotients involving fractional calculus operators. J. Math. Anal. 8(5), 47-70 (2017) 
8. Jakšetić, J., Pečarić, J.: Exponential convexity method. J. Convex Anal. 20(1), 181-187 (2013)

9. Keller, M., Pinchover, Y., Pogorzelski, F.: Optimal Hardy inequalities for Schrödinger operators on graphs. Commun. Math. Phys. 358(2), 767-790 (2018)

10. Khan, M.A., Pečarić, G., Pečarić, J.: On Zipf-Mandelbrot entropy. Comput. Appl. Math. 346, 192-204 (2019)

11. Mehmood, N., Agrwal, R.P., Butt, S.I., Pečarić, J.: New generalizations of Popoviciu-type inequalities via new Green's functions and Montgomery identity. J. Inequal. Appl. 2017, 108, 17 pages (2017)

12. Noor, M.A., Noor, K.I., Safdar, F.: Inequalities via generalized beta m-convex functions. J. Math. Anal. 9(3), 61-77 (2018)

13. Pečarić, J.: Connections among some inequalities of Gauss, Steffensen and Ostrowski. Southeast Asian Bull. Math 13(2), 89-91 (1989)

14. Pečarić, J., Kalamir, K.S., Varošanec, S.: Steffensen's and Related Inequalities (a Comprehensive Survey and Recent Advances). Monographs in Inequalities, vol. 7. Element, Zagreb (2014)

15. Pečarić, J., Praljak, M., Witkowski, A.: Linear operator inequality for $n$-convex functions at a point. Math. Inequal. Appl. 18, 1201-1217 (2015)

16. Pečarić, J., Proschan, F., Tong, Y.L.: Convex Functions, Partial Orderings and Statistical Applications. Academic Press, New York (1992)

17. Rabier, P.: Steffensen's inequality and $L^{1}-L^{\infty}$ estimates of weighted integrals. Proc. Am. Math. Soc. 140(2), 665-675 (2012)

18. Steffensen, J.F.: On certain inequalities between mean values, and their application to actuarial problems. Skand. Aktuarietidskr. 1, 82-97 (1918)

\section{Submit your manuscript to a SpringerOpen ${ }^{\circ}$ journal and benefit from:}

- Convenient online submission

- Rigorous peer review

- Open access: articles freely available online

- High visibility within the field

- Retaining the copyright to your article

Submit your next manuscript at $\gg$ springeropen.com 